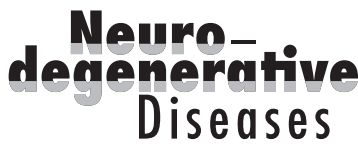

\title{
Function and Dysfunction of Presenilin
}

\author{
Jie Shen \\ Center for Neurologic Diseases, Brigham and Women's Hospital, Program in Neuroscience, Harvard Medical School, \\ Boston, Mass., USA
}

\section{Key Words}

Alzheimer's disease - Synapse - Neurodegeneration .

Presenilin $\cdot \gamma$-Secretase $\cdot$ Nicastrin $\cdot$ Notch

\begin{abstract}
The presenilin (PS) genes harbor approximately $90 \%$ of the identified mutations linked to familial forms of Alzheimer's disease, and the presenilin (PS) proteins are essential components of the $\gamma$-secretase complex involved in the proteolytic cleavage of type I receptors, such as Notch and the amyloid precursor protein. Genetic analysis employing cell type-specific conditional knockout technology highlighted the importance of PS in the adult brain, including learning and memory, synaptic function and age-dependent neuronal survival. In the central synapse, PS regulates neurotransmitter release, short- and long-term synaptic plasticity and calcium homeostasis. However, the molecular mechanisms by which PS maintains these essential functions are less clear. Although many $Y$-secretase substrates have been identified, their physiological relevance is often unclear. The findings that nicastrin and PS conditional knockout mice exhibit similar deficits in memory and age-dependent neurodegeneration are consistent with the notion that $\gamma$-secretasedependent activities of PS are required for the maintenance of memory and neuronal survival, though the $\gamma$-secretase physiological substrates, Notch receptors, are not targets of PS in the adult brain. Thus, despite of the intense interest in
\end{abstract}

\section{KARGER}

E-Mail karger@karger.com

www.karger.com/ndd
PS since its identification in 1995, more work is needed to define the molecular and cellular mechanisms by which PS controls brain functions and the dysfunction conferred by disease-causing mutations.

(c) 2013 S. Karger AG, Basel

Presenilins (PS1 and PS2) are the major causative genes of early-onset familial Alzheimer's disease (AD) and harbor more than 200 mutations, which represent approximately $90 \%$ of all identified mutations. Presenilins (PS) are essential components of $\gamma$-secretase, a multi-subunit protease complex that catalyzes the intramembranous cleavage of a number of type I transmembrane proteins, including Notch and the amyloid precursor protein. PS are essential for embryonic development, as PS1-/- mice die at birth and PS-/- mice die before embryonic day 9.5 [1-3]. During neural development, loss of PS results in the depletion of neural progenitor cells due to premature exit of cell cycle to differentiate into postmitotic neurons as well as neuronal migration defects, and PS regulates these processes primarily through the Notch signaling pathway $[1,4-7]$.

The embryonic lethality of PS germline knockout (KO) mice necessitated the employment of conditional gene target technology to develop conditional $\mathrm{KO}$ mice in which PS inactivation is restricted to specific cell types in the adult brain [8]. Using a specific aCaMKII-Cre 
transgenic line, we inactivated PS selectively in excitatory pyramidal neurons of the forebrain beginning at approximately postnatal day 18 [9]. Analysis of these PS conditional double $\mathrm{KO}(\mathrm{cDKO})$ mice at 2 months of age in the water maze and contextual fear conditioning paradigms revealed impaired spatial and associative memory [10]. Synaptic function is also selectively affected, as indicated by deficits in short- and long-term plasticity, such as synaptic facilitation and long-term potentiation (LTP), but use-dependent synaptic depression and long-term depression are unaffected [10-12]. Interestingly, presynaptic function (e.g., synaptic facilitation) is affected prior to postsynaptic defects (e.g., NMDA receptor-mediated responses), highlighting the importance of PS in the presynaptic terminal [12].

To determine the precise synaptic site of PS function, we further developed two unique lines of $\mathrm{cDKO}$ mice to restrict PS inactivation to hippocampal CA1 or CA3 neurons [11]. This strategy permitted identification of the effects of PS inactivation in either presynaptic or postsynaptic neurons of the Schaeffer collateral pathway. LTP and short-term plasticity are impaired after presynaptic but not postsynaptic deletion of PS, and the probability of evoked glutamate release is also reduced by presynaptic inactivation of PS. Thus, presynaptic inactivation of PS alone is sufficient to impair glutamatergic neurotransmitter release and LTP induction, even though NMDA receptor-mediated responses are normal. Interestingly, depletion of calcium internal stores by thapsigargin or inhibition of calcium release from these stores by ryanodine receptor inhibitors mimics and occludes the effects of presynaptic PS inactivation, suggesting that disrupted calcium homeostasis may underlie the presynaptic defects caused by PS inactivation [11, 13]. These findings also raise the possibility that presynaptic mechanisms may play a primary role in the pathophysiology of neurodegenerative disease [14], which is further supported by the presence of PS and $\gamma$-secretase substrates in presynaptic terminals $[11,15]$.

Strikingly, PS cDKO mice develop synaptic, dendritic and neuronal degeneration in an age-dependent manner with accompanying astrogliosis and hyperphosphorylation of tau, demonstrating an essential role for PS in neuronal survival $[10,16]$. The mechanism by which PS protects cortical neurons during aging is unique [17]. First, cortical neurons lacking PS appear to die via apoptosis, and the onset of apoptosis (about 2 months postnatally) is significantly delayed from the time of PS inactivation (about 1 month). Second, apoptosis occurs only in a tiny percentage (approximately $0.1 \%$ ) of these neurons during aging despite the fact that PS is inactivated in most if not all of these neurons. Over time, this low percentage of cell death rate can cumulatively lead to about 9 and $18 \%$ of neuron loss at 4 and 6 months of age, respectively [17]. By 6 months of age, these PS cDKO mice completely failed to learn the water maze and the fear conditioning tasks and exhibited behavioral deficits in the open field and the rotarod tests, suggesting that the significant loss of neurons is the likely cause for these severe behavioral phenotypes [10].

While the importance of PS in the promotion of neuronal survival during aging is unequivocal, the molecular target(s) by which PS protects cortical neurons has not been identified. The findings that conditional inactivation of nicastrin, another component of the $\gamma$-secretase complex, in the adult cerebral cortex similarly resulted in progressive memory impairment and neurodegeneration as in $P S \mathrm{cDKO}$ mice suggest that PS promotes memory and neuronal survival in a $\gamma$-secretase-dependent manner [18]. Although many substrates of $\gamma$-secretase have been reported [19], most of them were identified in overexpression systems and cell lines, so it is unclear how many of them are physiological substrates and whether $\gamma$-secretase-mediated cleavages of these substrates have physiological relevance. While Notch receptors appear to be key targets of PS during development $[4,5,20]$, conditional inactivation of Notch 1 and Notch 2 using the same aCaMKII-Cre transgenic line as we previously used to inactivate PS1 and nicastrin did not produce similar phenotypes as observed in PS $\mathrm{cDKO}$ and nicastrin $\mathrm{cKO}$ mice $[10,18,21]$. Furthermore, mRNA and protein levels of Notch1 and Notch2 are unaffected in the cerebral cortex of Notch 1 and Notch 2 cKO mice, respectively, indicating that Notch1 and Notch2 are not normally expressed in the excitatory pyramidal neurons of the cerebral cortex, which are selectively targeted by the aCaMKII-Cre transgenic line [21]. Thus, additional genetic efforts are needed to elucidate the molecular pathways underlying PSdependent neuronal survival.

Based on these in vivo findings and a large number of reports on the effects of familial $\mathrm{AD}$-linked mutations in culture and in vitro systems as well as in Caenorhabditis elegans, we previously proposed that PS mutations may cause dementia and neurodegeneration in $\mathrm{AD}$ via a partial loss-of-function mechanism [22]. Through the establishment of a sensitive cell culture system that lacks endogenous PS and allows quantitative assessment of $\gamma$-secretase activity from exogenous PS cDNAs, we found that all PS mutations impaired $\gamma$-secretase activity, and different mutations affected $\gamma$-secretase activity differentially [23]. One mutation in particular, L435F in the PAL 
motif, abolished $\gamma$-secretase activity completely, whereas the C410Y mutation had little residual activity [23]. Further in vivo analysis of these mutations through the generation of knockin mice would be informative to determine the consequence of these mutations under physiological conditions. The phenotypes of these mice will also be compared to another knockin mouse we recently developed, in which the $P S 1$ c.548G $>$ T mutation associated with frontotemporal dementia was introduced to examine the effects of this mutation [24]. Interestingly, this mutation, which resides at the boundary of exon and intron 6, causes exon skipping, and the aberrantly spliced products are degraded via nonsense-mediated decay, leading to decreased mRNA expression, though the corresponding G183V amino acid substitution does not affect $\gamma$-secretase activity [24].
In summary, our genetic studies have uncovered a novel loss-of-function pathogenic mechanism for AD and further suggested that defects in presynaptic neurotransmitter release may represent a convergent mechanism leading to neurodegeneration in affected circuits in neurodegenerative diseases. Therapeutic strategies directed toward restoring PS dysfunction may be effective in combating neurodegeneration in $\mathrm{AD}$.

\section{Acknowledgement}

The author would like to thank Adair Swain for her assistance. This work was supported by grants from the National Institute of Neurological Disorders and Stroke (NS041783, NS042818).

\section{References}

1 Shen J, Bronson RT, Chen DF, Xia W, Selkoe DJ, Tonegawa S: Skeletal and CNS defects in Presenilin-1-deficient mice. Cell 1997;89: 629-639.

2 Wong PC, Zheng H, Chen H, Becher MW, Sirinathsinghji DJ, Trumbauer ME, Chen HY, Price DL, Van der Ploeg LH, Sisodia SS: Presenilin 1 is required for Notch1 and DII1 expression in the paraxial mesoderm. Nature 1997;387:288-292.

3 Donoviel DB, Hadjantonakis AK, Ikeda M, Zheng H, Hyslop PS, Bernstein A: Mice lacking both presenilin genes exhibit early embryonic patterning defects. Genes Dev 1999;13: 2801-2810.

4 Wines-Samuelson M, Shen J: Presenilins in the developing, adult, and aging cerebral cortex. Neuroscientist 2005;11:441-451.

5 Handler M, Yang X, Shen J: Presenilin-1 regulates neuronal differentiation during neurogenesis. Development 2000;127:2593-2606.

6 Wines-Samuelson M, Handler M, Shen J: Role of presenilin-1 in cortical lamination and survival of Cajal-Retzius neurons. Dev Biol 2005;277:332-346.

7 Kim WY, Shen J: Presenilins are required for maintenance of neural stem cells in the developing brain. Mol Neurodegener 2008;3:2.

8 Beglopoulos V, Shen J: Gene-targeting technologies for the study of neurological disorders. Neuromolecular Med 2004;6:13-30.

-9 Yu H, Saura CA, Choi SY, Sun LD, Yang X, Handler M, Kawarabayashi T, Younkin L, Fedeles B, Wilson MA, Younkin S, Kandel ER, Kirkwood A, Shen J: APP processing and synaptic plasticity in presenilin-1 conditional knockout mice. Neuron 2001;31:713-726.

10 Saura CA, Choi SY, Beglopoulos V, Malkani S, Zhang D, Shankaranarayana Rao BS,
Chattarji S, Kelleher RJ 3rd, Kandel ER, Duff K, Kirkwood A, Shen J: Loss of presenilin function causes impairments of memory and synaptic plasticity followed by age-dependent neurodegeneration. Neuron 2004; 42:23-36.

11 Zhang C, Wu B, Beglopoulos V, Wines-Samuelson M, Zhang D, Dragatsis I, Sudhof TC, Shen J: Presenilins are essential for regulating neurotransmitter release. Nature 2009;460: 632-636.

12 Zhang D, Zhang C, Ho A, Kirkwood A, Sudhof TC, Shen J: Inactivation of presenilins causes pre-synaptic impairment prior to postsynaptic dysfunction. J Neurochem 2010;115: 1215-1221.

13 Ho A, Shen J: Presenilins in synaptic function and disease. Trends Mol Med 2011;17:617624.

14 Shen J: Impaired neurotransmitter release in Alzheimer's and Parkinson's diseases. Neurodegener Dis 2010;7:80-83.

15 Saura CA, Chen G, Malkani S, Choi SY, Takahashi RH, Zhang D, Gouras GK, Kirkwood A, Morris RG, Shen J: Conditional inactivation of presenilin 1 prevents amyloid accumulation and temporarily rescues contextual and spatial working memory impairments in amyloid precursor protein transgenic mice. J Neurosci 2005;25:6755-6764.

-16 Beglopoulos V, Sun X, Saura CA, Lemere CA, Kim RD, Shen J: Reduced beta-amyloid production and increased inflammatory responses in presenilin conditional knock-out mice. J Biol Chem 2004;279:46907-46914.

17 Wines-Samuelson M, Schulte EC, Smith MJ, Aoki C, Liu X, Kelleher RJ 3rd, Shen J: Characterization of age-dependent and progressive cortical neuronal degeneration in prese- nilin conditional mutant mice. PLoS One 2010;5:e10195.

18 Tabuchi K, Chen G, Sudhof TC, Shen J: Conditional forebrain inactivation of nicastrin causes progressive memory impairment and age-related neurodegeneration. J Neurosci 2009;29:7290-7301.

19 Haapasalo A, Kovacs DM: The many substrates of presenilin/gamma-secretase. J Alzheimers Dis 2011;25:3-28.

20 Song W, Nadeau P, Yuan M, Yang X, Shen J, Yankner BA: Proteolytic release and nuclear translocation of Notch-1 are induced by presenilin-1 and impaired by pathogenic presenilin-1 mutations. Proc Natl Acad Sci USA 1999;96:6959-6963.

21 Zheng J, Watanabe H, Wines-Samuelson M, Zhao H, Gridley T, Kopan R, Shen J: Conditional deletion of Notch1 and Notch2 genes in excitatory neurons of postnatal forebrain does not cause neurodegeneration or reduction of Notch mRNAs and proteins. J Biol Chem 2012;287:20356-20368.

22 Shen J, Kelleher RJ 3rd: The presenilin hypothesis of Alzheimer's disease: evidence for a loss-of-function pathogenic mechanism. Proc Natl Acad Sci USA 2007;104:403-409.

23 Heilig EA, Xia W, Shen J, Kelleher RJ 3rd: A presenilin-1 mutation identified in familial Alzheimer disease with cotton wool plaques causes a nearly complete loss of gammasecretase activity. J Biol Chem 2010;285: 22350-22359.

24 Watanabe H, Xia D, Kanekiyo T, Kelleher RJ 3rd, Shen J: Familial frontotemporal dementia-associated presenilin-1 c.548G $>\mathrm{T}$ mutation causes decreased mRNA expression and reduced presenilin function in knock-in mice. J Neurosci 2012;32:5085-5096. 